\title{
Dependability in Cyber-Physical Systems and Applications
}

\author{
MD ZAKIRUL ALAM BHUIYAN, Fordham University, USA \\ SY-YEN KUO, National Taiwan University, Taiwan \\ DAMIAN LYONS, Fordham University, USA \\ ZILI SHAO, The Hong Kong Polytechnic University, Hong Kong
}

CCS Concepts: • Computer systems organization $\rightarrow$ Embedded and cyber-physical systems; Dependable and fault-tolerant systems and networks;

Additional Key Words and Phrases: Cyber-physical systems and applications, dependability, reliability, security and privacy, distributed systems

\section{ACM Reference format:}

Md Zakirul Alam Bhuiyan, Sy-Yen Kuo, Damian Lyons, and Zili Shao. 2018. Dependability in Cyber-Physical Systems and Applications. ACM Trans. Cyber-Phys. Syst. 3, 1, Article 1 (September 2018), 4 pages.

https://doi.org/10.1145/3271432

\section{INTRODUCTION}

Cyber-systems are now available anytime, anywhere, by different means, and distributed unobtrusively throughout everyday environments in which physical objects/artifacts embedded with invisible computers are sensible and networked locally and globally. The Cyber-Physical System (CPS) is a speedily emerging arena, which is going to cover every aspect of life in the near future. Semiconductors and the Internet have revolutionized and transformed our lives in how we interact with information and led to the growth of information technology; now we are into a paradigm of CPS that would transform the way we interact with and manipulate the physical systems. As CPS represents a bold new generation of systems that integrate computing and communication capabilities with the dynamics of physical and engineered systems, it has not been realized how it is going to impact the economy and social structure as vast major investments are being made worldwide in developing this technology. The physical world, however, is not entirely predictable. Although it is yet unclear what exactly the real cyber-physical integrated worlds would be, there is no doubt that they must be "dependable" (Bhuiyan et al. 2016, 2017a).

Increasingly, individuals, practitioners, and organizations started developing or procuring sophisticated CPSs on whose dependability of services they need to place great confidence. Future CPSs need to close the dependability gap in the face of challenges in different circumstances and services, e.g., continuity, effective performance, real-time responsiveness, ability to overcome data

Authors' addresses: M. Z. A. Bhuiyan and D. Lyons, Department of Computer and Information Sciences, Fordham University, New York, NY 10458; emails: \{mbhuiyan3, dlyons\}@fordham.edu; S.-Y. Kuo, Department of Electrical Engineering, National Taiwan University, Taipei, Taiwan 10617; email: sykuo@cc.ee.ntu.edu.tw; Z. Shao, Department of Computing, Hong Kong Polytechnic University, Hung Hom, Hong Kong; email: cszlshao@comp.polyu.edu.hk.

Permission to make digital or hard copies of part or all of this work for personal or classroom use is granted without fee provided that copies are not made or distributed for profit or commercial advantage and that copies bear this notice and the full citation on the first page. Copyrights for third-party components of this work must be honored. For all other uses, contact the owner/author(s).

2018 Copyright is held by the owner/author(s).

2378-962X/2018/09-ART1

https://doi.org/10.1145/3271432 
fault/corruption/anomaly, ability to avoid catastrophic failures, prevention of deliberate privacy intrusions, reliability/availability/sustainability/adaptability/heterogeneity/security/safety, and so on (Zeng et al. 2017; Bhuiyan et al. 2017b). Therefore, we have a wide open area of research to explore and exploit the challenges and immense opportunities in this dependable CPS arena.

\section{ARTICLE COLLECTION AND SELECTION}

The special issue on dependability in cyber-physical systems and applications is motivated by the compelling challenges and requirements identified above, and aims to compile research that fundamentally addresses them. ACM TCPS has recognized the important and timely concerns in this special issue. It has attempted to provide an opportune forum for presenting current research ideas, techniques, and the like from researchers and practitioners belonging to myriad research areas, with the final goal of sharing their specific challenges and solutions for CPS dependability. We received an overwhelming response from the research community. Particularly, we received a total of 64 submissions. After a rigorous review process, a total of 11 articles were accepted for publication, making an attractive acceptance rate of $17.18 \%$.

The selected articles contributed to the important advances toward CPS dependability issues. The contributions cover several topics, which can be grouped into three different and distinct categories: (1) dependable CPS control and performance evaluation, (2) dependability in localization and smart grid topology, and (3) dependability in data collection and analytics. All of the 11 articles were contributed by researchers from both academia and industry.

\section{LIST OF CONTRIBUTIONS TO THE SPECIAL ISSUE}

After a rigorous review process, the following 11 contributions have been considered:

(1) Cross-Domain Noise Impact Evaluation for Black Box Two-Level Control CPS

(2) Holistic Cyber-Physical Management for Dependable Wireless Control Systems

(3) Resource Cost-Aware Fault-Tolerant Design Methodology for End-to-End Functional Safety Computation on Automotive Cyber-Physical Systems

(4) Dependable Visual Light-Based Indoor Localization with Automatic Anomaly Detection for Location-Based Service of Mobile Cyber-Physical Systems

(5) Improve the Localization Dependability for Cyber-Physical Applications

(6) A Dependable Time Series Analytics Framework for Cyber-Physical Systems of IoTBased Smart Grid

(7) Privacy-Preserving Data Aggregation Computing in Cyber-Physical Social Systems

(8) Securing Communication Data in Pervasive Social Networking Based on Trust with $\mathrm{KP}-\mathrm{ABE}$

(9) On Reliability Analysis of Smart Grids under Topology Attacks: A Stochastic Petri Net Approach

(10) Dependable Deep Computation Model for Feature Learning on Big Data in CyberPhysical Systems

(11) Dependable Model-Driven Development of CPS: From Stateflow Simulation to Verified Implementation

\section{SUMMARY OF THE CONTRIBUTIONS}

The first group of contributions (dependable CPS control and performance evaluation) includes four articles. The first article of this group focused on two-level control CPSs, a widely adopted control CPS architecture, and proposed an emulation-based evaluation methodology framework. The framework uses hybrid model reachability to quantify cross-domain noise impact and exploits 
Lyapunov stability theories to reduce the evaluation benchmark size. To enhance the dependability of industrial wireless control, in the second of this group, Ma et al. proposed a holistic cyberphysical management framework that employs runtime coordination between the plant control and network management. The design includes a holistic controller that generates actuation signals to physical plants and reconfigures the wireless sensor and actuator networks (WSANs) to maintain desired control performance while saving wireless resources. The third article of the group contributed to the resource cost-aware fault-tolerant design methodology for end-to-end functional safety computation on automotive CPSs. The last article of the group is about dependable model-driven development of CPSs, which was proposed by Yu et al.

The second group of contributions (dependability in localization and smart grid topology) includes three articles. Liu et al. developed a dependable visual light-based indoor localization framework. A method is derived from the triangle inequality to automatically detect the abnormal LED lamps that are blocked by obstacles. These LED lamps are then ignored by their localization algorithm so that they do not bias the localization results, which improves the dependability of the localization framework. Tian et al. proposed to exploit the localized users as the mobile anchors for localizing the nonlocalized users in the CPSs. These mobile users cooperate as a whole group to improve their localization accuracy. For the scenarios with a crowd of users, the article divides the users into different groups according to their distance information, which can ensure that only the dependable anchors are used for the localization. The last article of the group proposed by Beibei et al. developed a stochastic Petri-net-based analytical model to assess and analyze the system reliability of smart grid CPSs, specifically against topology attacks, and system countermeasures (i.e., intrusion detection systems and malfunction recovery techniques).

The final group of contributions (dependability in data collection and analytics) includes four articles. Chang et al. proposed DTSA, a dependable time series data analytics framework, which is for the IoT-based smart grid CPS. The DTSA framework is capable of proving dependable data transforming from the CPS to the target database with an extraction engine to preliminarily refine raw data and further cleanse the data with a correction engine. The second article of this group proposed by Qingchen et al. suggested a dependable deep computation model for feature learning on low-quality big data in cyber-physical systems. Specially, a regularity is added into the objective function of the deep computation model to obtain reliable features in the intermediate-level representation space in the CPS. Next, Kun et al. utilized a bitwise XOR and presented a bit-choosing algorithm to realize privacy-preserving $\mathrm{min}, k$ th $\mathrm{min}$, and percentile computation. The last article of the group proposed by Ning et al. developed a scheme to guarantee communication data security in PSN based on two dimensions of trust in a flexible manner on the basis of Key-Policy Attribute-Based Encryption (KP-ABE). Its advantages and performance are justified and evaluated through extensive analysis on security, computation complexity, communication cost, scalability, and flexibility, as well as scheme implementation.

\section{CONCLUSIONS AND ACKNOWLEDGMENTS}

Editing this special issue has been an advanced and valuable experience to us. Although the selected articles contribute and address only a limited set of issues related to the dependability in cyber-physical systems and applications, they clearly demonstrate how a lack of dependability in systems and applications can influence overall system and application performance. We would like to thank all the researchers who responded to the call and acknowledge the tremendous efforts that went into their submissions. Also, we express sincere gratitude to the reviewers for providing high-quality reviews in a timely fashion to enhance the quality of the accepted articles. Finally, we would like to thank Professor Tei-Wei Kuo, the editor-in-chief of the ACM Transactions on Cyber-Physical Systems, and his editorial team and support team for their consistent support on 
this special issue. We hope that the readers will enjoy the articles picked for this special issue and that it will facilitate further research in this exciting area.

\section{REFERENCES}

Md Zakirul Alam Bhuiyan, Jie Wu, Guojun Wang, Jiannong Cao, Wenjun Jiang, and Mohammed Atiquzzaman. 2017a. Towards cyber-physical systems design for structural health monitoring: Hurdles and opportunities. ACM Trans. CyberPhys. Syst. 1, 4, Article 19 (2017), 26 pages. DOI : https://doi.org/10.1145/3086508

Md Zakirul Alam Bhuiyan, Guojun Wang, and Athanasios V. Vasilakos. 2017b. Dependability in parallel and distributed systems and applications. Inform. Sci. 329, 1 (2017), 1-2.

Md Zakirul Alam Bhuiyan, Jie Wu, Guojun Wang, and Jiannong Cao. 2016. Sensing and decision-making in cyber-physical systems: The case of structural health monitoring. IEEE Trans. Industr. Inform. 12, 6 (2016), 2103-2114.

Jing Zeng, Laurence T. Yang, Man Lin, Zili Shao, and Dakai Zhu. 2017. The system-level design optimization for securitycritical cyber-physical-social systems. ACM Trans. Embed. Comput. Syst. 16, 39 (2017), 1-39.

Received June 2018; revised July 2018; accepted August 2018 